\title{
Modeling blast loading on buried reinforced concrete structures with Zapotec
}

\author{
Greg C. Bessette* \\ Sandia National Laboratories, P.O. Box 5800, Albuquerque, NM 87185-0378, USA
}

Received 18 January 2005

Revised 1 February 2007

\begin{abstract}
A coupled Euler-Lagrange solution approach is used to model the response of a buried reinforced concrete structure subjected to a close-in detonation of a high explosive charge. The coupling algorithm is discussed along with a set of benchmark calculations involving detonations in clay and sand.
\end{abstract}

\section{Introduction}

Modeling the response of buried reinforced concrete (RC) structures subjected to close-in detonations of conventional high explosives poses a challenge for a number of reasons. Foremost, there is the potential for coupled interaction between the blast and structure. Coupling enters the problem whenever the structure deformation affects the stress state in the neighboring soil, which in turn, affects the loading on the structure. For close-in detonations, coupled interaction is generally assured since the induced loading usually results in significant structural deformations. Additional challenges for numerical modeling include handling disparate degrees of material deformation encountered in the structure and surrounding soil, modeling the structure details (e.g., modeling the concrete with embedded reinforcement, jointed connections, etc.), providing adequate mesh resolution, and characterizing the soil response under blast loading.

There are numerous numerical approaches for modeling this class of problem (e.g., see Mair [7] for a review of applicable approaches). The focus of this work will be the use of a coupled Euler-Lagrange (CEL) solution approach. In particular, the development and application of the CEL capability within the Zapotec code is described. A CEL approach is well suited for modeling blast/structure interaction as it allows flexibility in handling different portions of the problem using either Eulerian or Lagrangian techniques. For example, the explosive and soil can be modeled as Eulerian as this approach readily handles the shock transmission and large material deformations involved. The $\mathrm{RC}$ structure can be modeled using a Lagrangian finite element (FE) method as this allows for detailed modeling of structure components and their response.

The application of the CEL methodology within Zapotec for modeling coupled blast/structure interaction will be investigated by a series of benchmark calculations. These benchmarks rely on data from the Conventional Weapons Effects Backfill (CONWEB) test series [6]. In these tests, a 7-kg pipe-encased C-4 charge was detonated in soil at a $1.52 \mathrm{~m}$ standoff from a buried test structure. The structure was composed of a RC slab bolted to a reaction structure. Both the slab thickness and soil media were varied in the test series. The wealth of data obtained from these tests along with the variations in experimental setups provides ample opportunity to assess the robustness of the CEL methodology.

\footnotetext{
*Corresponding author. E-mail: gcbesse@ sandia.gov.
} 


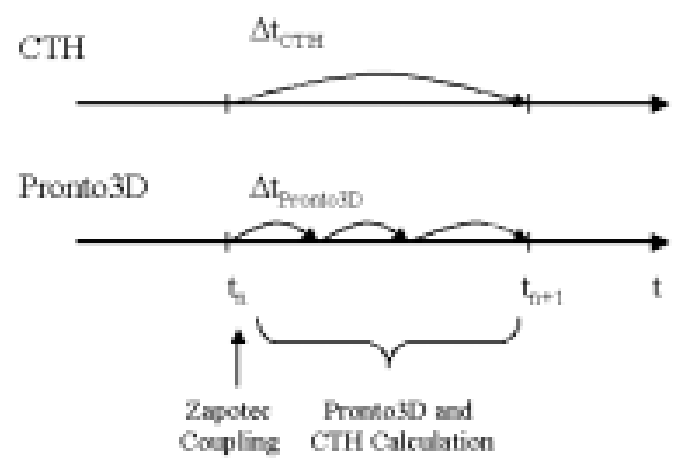

Fig. 1. Time synchronization of CTH and Pronto3D.

\section{The coupling algorithm}

Zapotec links the CTH and Pronto3D codes. CTH, a shock physics code, performs the Eulerian portion of the calculation, while Pronto3D, an explicit FE code, performs the Lagrangian portion. The two codes are run concurrently with the appropriate portions of a problem solved on their respective computational domains. Zapotec handles the coupling between domains. Both CTH and Pronto3D are well documented (e.g., see Bell et al. [5] and Attaway et al. [1]). The remaining discussion will focus on CEL methodology within Zapotec.

Zapotec controls both the time synchronization between CTH and Pronto3D as well as the interaction between materials on their respective domains. The time synchronization is illustrated in Fig. 1. At a given time $t_{n}$, Zapotec performs the coupled treatment. Once this treatment is complete, both CTH and Pronto3D are run independently over the next Zapotec time step. In general, the Pronto3D stable time step will be smaller than that for CTH. When this occurs, Zapotec allows subcycling of Pronto3D for computational efficiency. The subcycling continues until time $t_{n+1}$ is reached, ensuring the two codes are synchronized.

The coupling at time $t_{n}$ involves getting data from CTH and Pronto3D, working on the data, then passing the updated data back to the two codes. Zapotec first operates on the CTH data, a process termed material insertion. This involves mapping the current configuration (and state) of a Lagrangian body onto the fixed Eulerian mesh. The insertion algorithm determines what portions of a Lagrangian body are overlapping the CTH mesh. State data from the overlapping Lagrangian body are then mapped into the CTH mesh. Mapped data include the mass, momentum, stress, sound speed, and internal energy. In general, a CTH cell will be overlapped by multiple Lagrangian elements. When this occurs, the mapped element quantities are weighted by their volume overlap. The weighted quantities are accumulated for all elements overlapping a cell, after which the intrinsic value is recovered for insertion. The inserted data are then passed back to CTH as an update.

Once the material insertion is complete, the external loading on a Lagrangian material surface is determined from the stress state in the neighboring Eulerian material. Since the surface is uniquely defined, it is straightforward to determine the external force on a surface element from the traction vector, element surface normal, and area. After processing each surface element, the element-centered forces are distributed to the nodes and passed back to Pronto3D as a set of external nodal forces. Once the coupled treatment is complete, both CTH and Pronto3D are run independently over the next time step with their updated data.

\section{CONWEB test description}

Waterways Experiment Station conducted a series of tests, referred to as the Conventional Weapon Effects Backfill (CONWEB) tests, to develop a consistent set of ground shock and structural response data for explosive charges detonated in different soil backfills [6]. In these tests, a 7-kg pipe-encased, C-4 charge was emplaced $1.52 \mathrm{~m}$ from the structure, which was composed of a RC slab bolted to a reaction structure (see Fig. 2). Both the structure and surrounding soil were instrumented. Tests 1 through 3 will be modeled with Zapotec. The first two tests were 


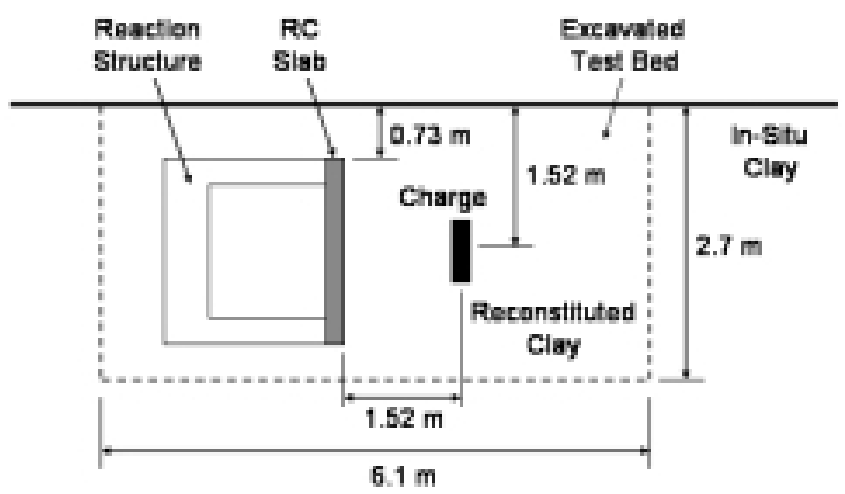

Fig. 2. Test setup.

conducted in reconstituted clay. The third was conducted in compacted concrete sand. The average wet density and air void content of the clay was $1962 \mathrm{~kg} / \mathrm{m}^{3}$ and 4.0 percent, respectively. For the sand, they were $1865 \mathrm{~kg} / \mathrm{m}^{3}$ and 25.3 percent, respectively.

Details regarding the test setup and structure construction can be found in Hayes [6]. Further details regarding this analysis can be found in Bessette [4]. Only a summary is provided here. The RC slab was $4.57 \mathrm{~m}$ long and $1.65 \mathrm{~m}$ high. The slab thickness and reinforcement were varied in the tests. In Test 1 and 3, the slab thickness and reinforcement ratio was $10.9 \mathrm{~cm}$ and 1.0 percent, respectively. In Test 2, they were $21.8 \mathrm{~cm}$ and 0.5 percent, respectively. The unconfined compressive strength of the slab on the day of the tests were 42.0, 44.1, and $40.4 \mathrm{MPa}$ for the three tests, respectively. Prior to a test, the RC slab was bolted to the reusable reaction structure and placed in an excavated test bed, which was then backfilled with soil. Care was taken during backfilling to ensure consistent soil properties throughout the test bed. The explosive charge was placed $1.52 \mathrm{~m}$ from the surface of the slab at a depth of $1.52 \mathrm{~m}$. For Tests 1 and 3, the charge was vertically oriented as depicted in Fig. 2. For Test 2, the charge was oriented horizontally.

\section{Analysis of test 1}

The problem setup is depicted in Fig. 3. The problem is composed of three components: the explosive charge, soil, and structure. The charge and soil were modeled as Eulerian, while the structure was modeled as Lagrangian. The CTH mesh encompassed the charge and structure, having a nominal resolution of $3 \mathrm{~cm}$. Soil was inserted throughout the CTH mesh, with void specified above the soil surface and within the structure's interior volume. Symmetry planes were assumed along the mid-length of the structure and along the cased charge to reduce the problem size (i.e., along the $x=0$ and $y=0$ planes). The latter is a non-physical plane of symmetry. Preliminary analyses examined this choice by including the free-field region in the calculation (see Bessette [4] for details). Modeling the free-field region appeared to have little effect on the calculated results, at least over the range of the measured data $(\sim 20 \mathrm{msec})$. The assumption of a symmetry plane about the explosive will break-down at later times; however, this was not an issue here since the calculations were only carried out to the extent of the available accelerometer and interface pressure data.

The CTH mesh used in these calculations contained approximately 1.7 million cells. It should be noted that the chosen mesh resolution is inadequate to properly model the detonation process within the explosive charge; however, the coarseness of the mesh does not appear to have affected the pressure field in the soil away from the explosive source (i.e., there was good correlation of the calculated and measured pressures at standoffs coincident with the structure location).

CTH decouples the material behavior into its dilatational and deviatoric response. The dilatational response is described by an equation of state (EOS), while a strength model describes the deviatoric response. The definition of material properties for the explosive charge and steel case were straight-forward, where the CTH material library 


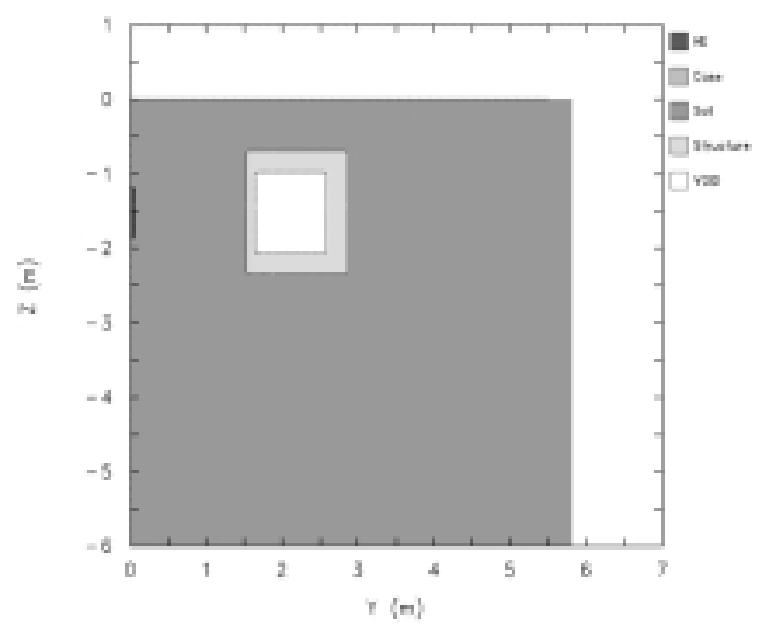

(a) Side View

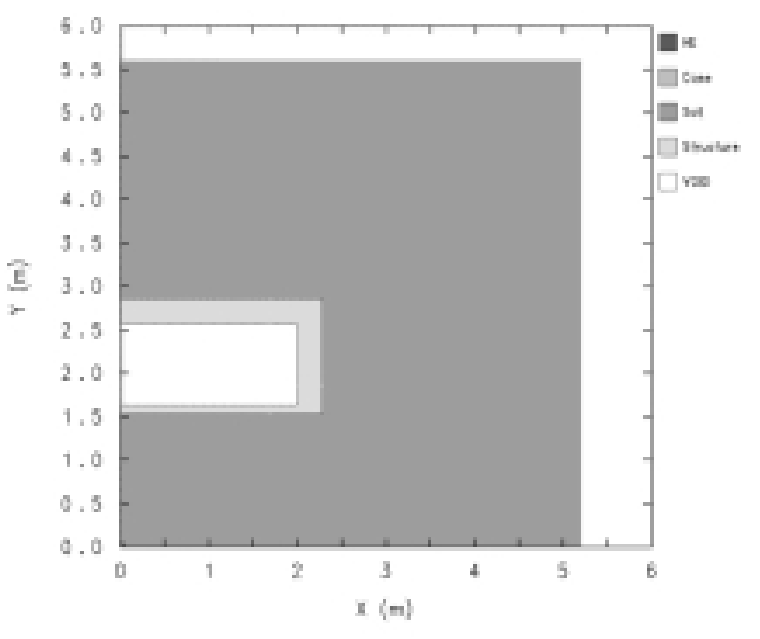

(b) Top View

Fig. 3. Problem setup

JWL EOS for C-4 was used to model the explosive and the case was modeled as a mild steel. The programmed burn option was specified for the explosive, with initiation assumed at the charge centroid. Development of a constitutive relationship for the clay was not so straight-forward. Baylot [3] reported material fits to the static uni-axial strain and tri-axial compression data. These fits were used as a basis in the development of a material model for CTH, where the dilatational response was modeled using a P-alpha EOS and the geologic (GEO) model was used for the deviatoric response. The P-alpha EOS is commonly used to model porous materials, where alpha is a state variable that tracks the crushing of the pores until the fully dense material response is recovered. The GEO model admits a pressure-dependent yield surface.

A multi-step approach was taken in the development of the material model. First, the prescribed deformation (PRDEF) option in CTH was used to develop an initial fit to the static material data. Once a reasonable fit was obtained, a series of CTH calculations that did not include the structure were conducted to draw comparisons with the measured free-field impulse and velocity data. Free-field data were collected at standoffs ranging from 0.91 to $2.13 \mathrm{~m}$ at $0.30 \mathrm{~m}$ intervals, with the gage data used for comparisons residing along a radial axis emanating from the center of the charge. These comparisons provided an independent means for model validation. During the course of model evaluation, it was found that use of the initial fit lead to severe over-predictions in the free-field velocities. The cohesion, initial slope at zero pressure, and ultimate yield in the GEO model were adjusted to add strength to the material in order to bring the calculated free-field velocities more in-line with the measured data. Figure 4 provides comparisons of the CTH-developed fits and those reported in Baylot [3]. The calibrated fit exhibits significant strength enhancement at higher pressures. The difference in strength appears significant on the scale of the plot; however, the enhanced strength is quite small when compared with other media such as sand. One should bear in mind that the calibrated model is tuned to the measured free-field data and is only meant to provide an approximation of the dynamic soil response.

A FE model of the structure was developed (see Fig. 5), which explicitly modeled the reinforcement and bolted connections using 2-node beam elements. The concrete was modeled using 8-node uniform strain hexahedral elements, having a nominal resolution of $1.9 \mathrm{~cm}$. There were approximately 80,000 elements in the mesh.

Pronto3D's rebar model [2] was used to represent the behavior of the reinforcement. This model includes both hardening and rate effects. The concrete response was modeled using the Karagozian and Case (K\&C) model [2,8]. This model can be described as a concrete plasticity model, which decouples the dilatational and deviatoric response. A tabulated EOS defines the pressure as a function of the current and previous minimum volumetric strains. The deviatoric portion of the model admits three yield surfaces: the yield failure surface, the maximum failure surface, and the residual failure surface. These failure surfaces serve to track the damage evolution within the concrete. 


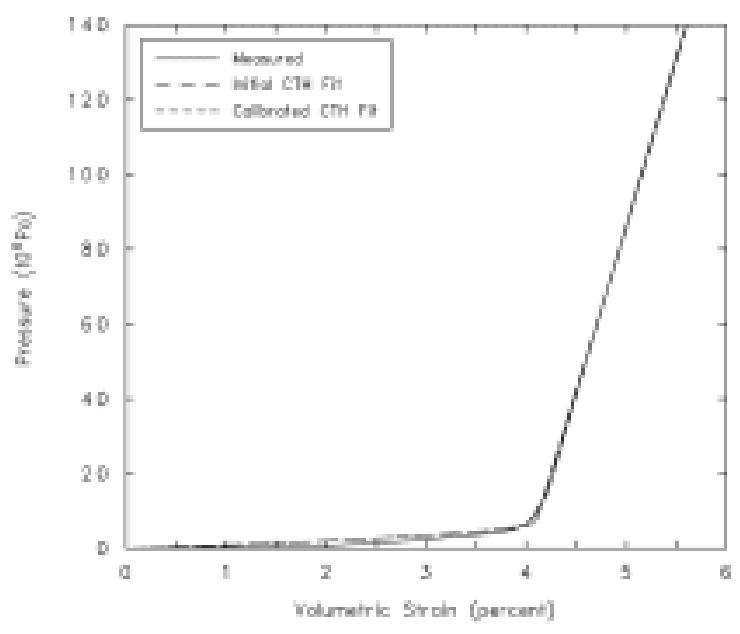

(a) Hydrostatic

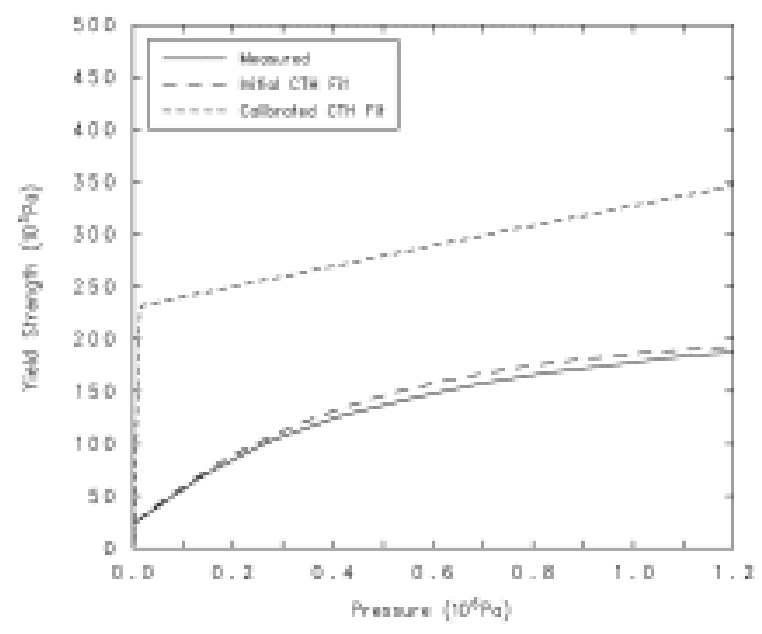

(b) Yield Envelope

Fig. 4. Material response for clay.

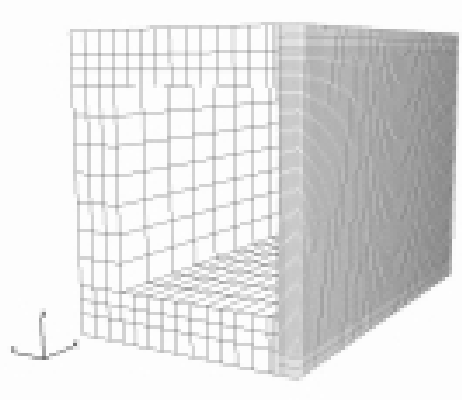

(a) Overall Mesh

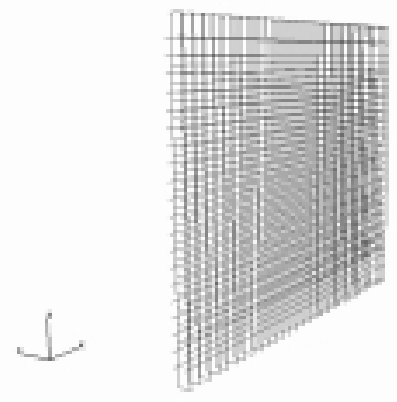

(b) Slab Reinforcement

Fig. 5. FE model of the structure.

There are many versions of the K\&C concrete model. The version implemented into Pronto3D allows the user to input a few selected material parameters (unconfined compressive strength, density, Poisson ratio, fractional dilatancy, and maximum aggregate size) which are then used by the model to automatically develop additional material data used internally by the code (e.g., determination of a strain rate enhancement factor, which is derived from the unconfined compressive strength). Caution should be exercised when using an automated model since no two concretes are exactly alike. For this analysis, there was little choice since only the unconfined compressive strength was available for the concrete used in the CONWEB tests.

In the Zapotec problem setup, the relationship between Eulerian and Lagrangian materials must be defined. This relationship includes a definition of Lagrangian materials that will be mapped into the CTH mesh as well as identifying Lagrangian surfaces that can interact with neighboring Eulerian materials. For this analysis, the concrete in the structure is mapped into the CTH mesh and the structure's exterior surface is defined as an Eulerian contact surface.

The detonation of the explosive charge results in a shock being transmitted into the soil. Following the initial shock transmission, a cavity is formed in the soil which is composed of expanding gaseous explosive products. The cavity expands at a much slower rate as compared with the shock velocity. By $2 \mathrm{msec}$, the shock reaches the structure and it begins to respond. By $4 \mathrm{msec}$, there is noticeable deformation of the structure, which continues over time (see Fig. 6). The combination of slab thinness and high shock transmission qualities in the clay results in significant slab 


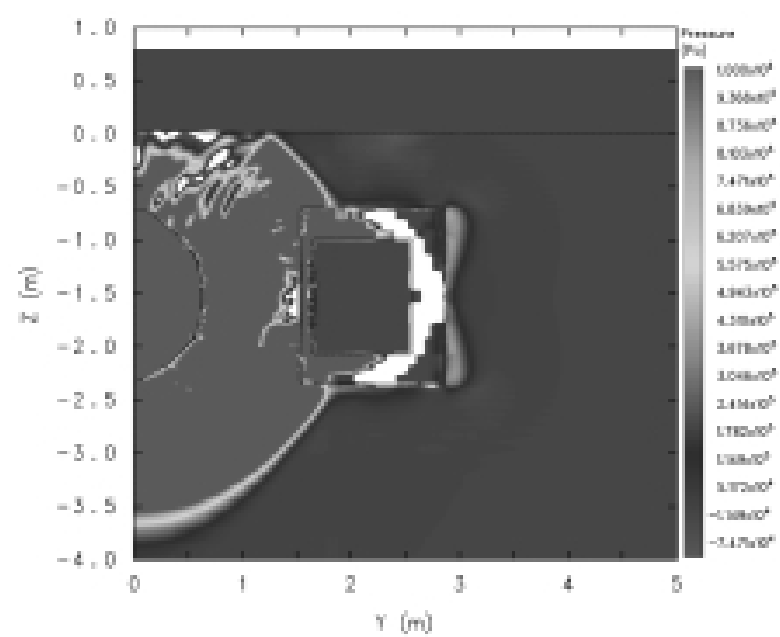

(a) Pressure Plot at $4 \mathrm{msec}$

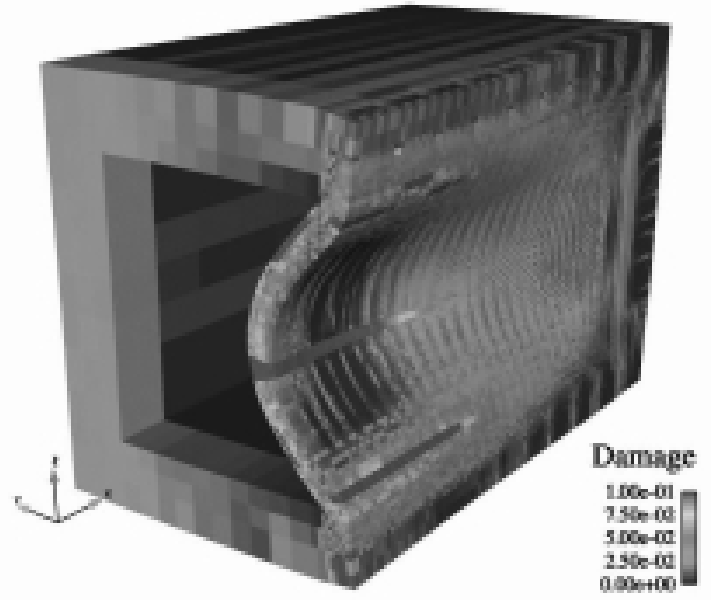

(b) Deformed Mesh at $20 \mathrm{msec}$

Fig. 6. Typical results.

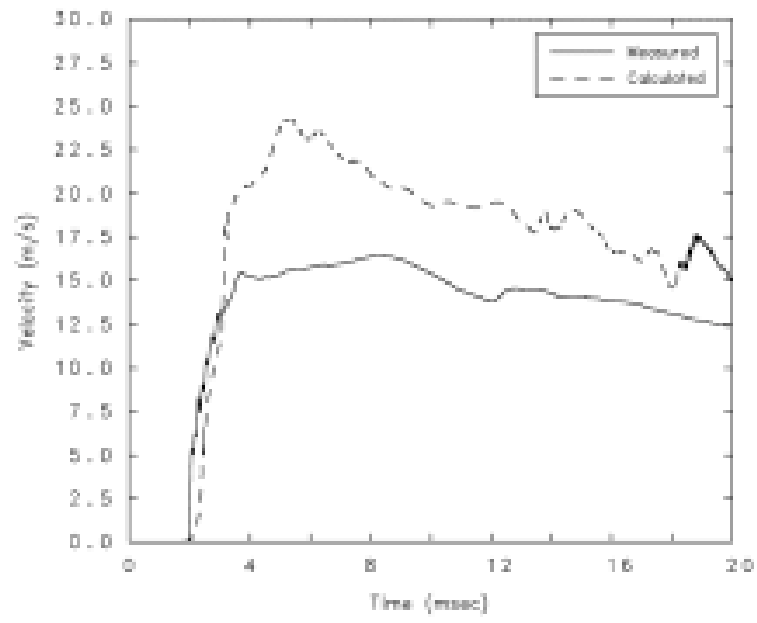

(a) AHS-0

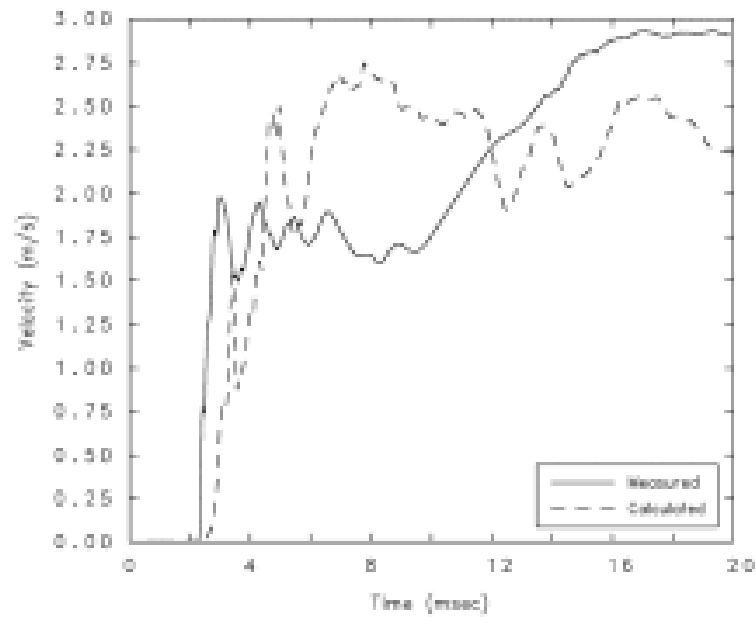

(b) AHS-10

Fig. 7. Velocity at accelerometer locations AHS-0 and AHS-10, Test 1.

deformation. In addition, the structure undergoes a rigid body motion, resulting in an interface loading arising at the rear of the structure (e.g., see Fig. 6(a)). The latter is a consequence of soil resistance to the structure's rigid body motion.

The calculation was run for $20 \mathrm{msec}$, the extent of the available test data. The analysis suggests the slab will be breached. The size of the breach could not be determined; however, it was evident there would be extensive damage along the slab center as well as at the end supports near the structure centerline (see Fig. 6(b)). This is consistent with the damage observed in the test.

Comparisons were drawn with the measured velocity at selected accelerometer locations in the structure. For brevity, only the comparisons at locations AHS-0 and AHS-10 are shown (see Fig. 7). Location AHS-0 resides at the interior surface of the slab directly opposite the charge centroid. Location AHS-10 resides on the interior surface at the base of the reaction structure, offset $0.46 \mathrm{~m}$ from the slab. These accelerometers measured the horizontal motion 


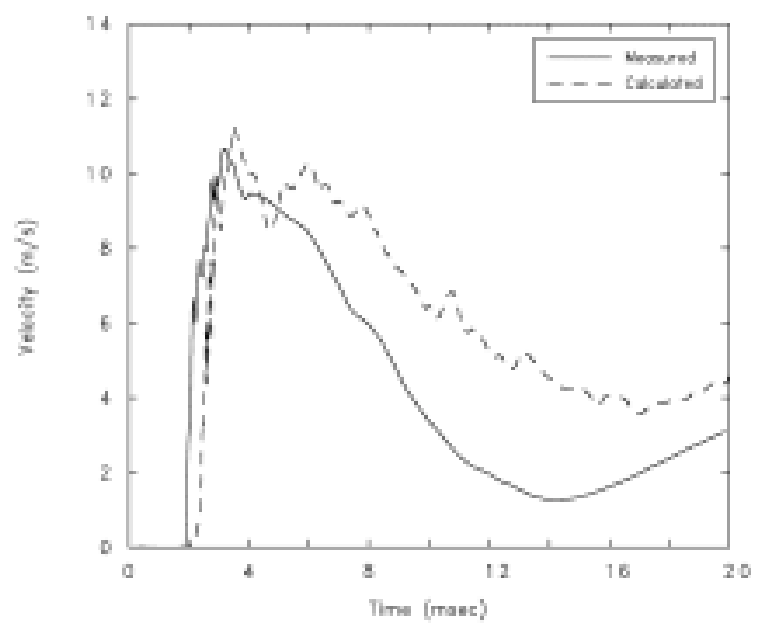

(a) AHS-0

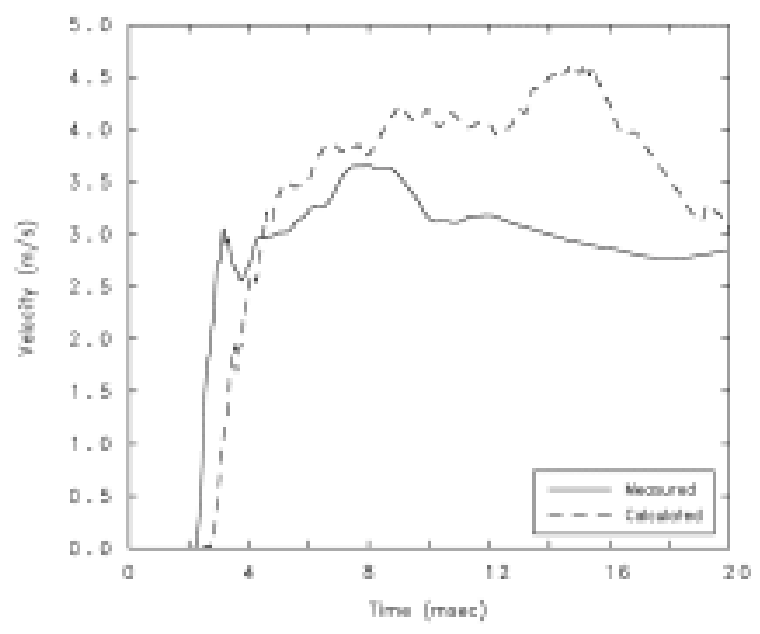

(b) AHS-10

Fig. 8. Velocity at accelerometer locations AHS-0 and AHS-10, Test 2.

of the structure. Care should be exercised with interpreting the accelerometer data. There is limited confidence in the measured late-time velocity for the slab. The primary reason is that the structure was breached and it is not known when the accelerometers became detached from the slab. However, the measured peak velocity is thought to be reliable. As shown in Fig. 7(a), the slab velocity at AHS-0 is over-predicted, with these results typical of those calculated at other slab locations. Good correlation with the data was noted for gages located in the reaction structure, suggesting Zapotec is providing a good approximation of the loading on the rear surface of the structure.

The reason for the velocity over-prediction is largely attributed to inaccuracies in soil modeling. The soil model was developed from limited static material data, and calibrated to measured free-field impulse and velocity data. Accordingly, one can only expect an approximation of the dynamic soil response. This conclusion was tested by a parameter study to assess uncertainties associated with modeling the structure (e.g., addressing the influence of mesh resolution, constitutive modeling of both the concrete and reinforcement) and soil response. Variations in soil modeling exhibited a first-order effect on the analysis, with other parameter variations having a much smaller effect on the results. The parameter study is discussed in detail in [4].

\section{Analysis of test 2}

The problem setup for Test 2 was comparable to Test 1, with changes made to the FE model to account for the thicker RC slab and the charge orientation modified to a horizontal configuration. The physics of the event were generally comparable to that noted in Test 1 , with the exception that there was significantly less deformation of the slab. The calculation indicated higher loads on the structure with a greater degree of rigid body motion, which is consistent with the measured data. Also, the calculated damage to the RC slab could be characterized as light-to-moderate, which is consistent with that observed in the test.

Comparisons of the structure velocities at AHS-0 and AHS-10 are provided in Fig. 8. One notes excellent correlation with the measured peak velocity, with a tendency to over-predict the velocity at later times. These results are typical of those noted at other gage locations. The calculation was run to $90 \mathrm{msec}$ to assess the later-time structural response. The calculated permanent displacement at the slab center was approximately $3.6 \mathrm{~cm}$. This value was derived from the nodal history data by taking the relative displacement between the reaction structure and data at AHS-0. Review of the nodal history data and deformed mesh plots indicated the 90 msec duration was sufficient for the purposes of determining the permanent displacement. The calculated displacement compared well with the measured value of $3 \mathrm{~cm}$. 


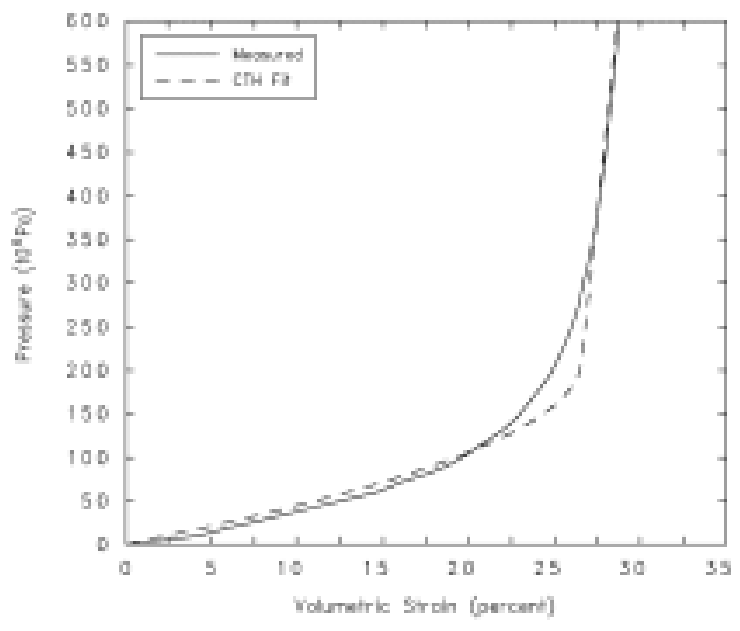

(a) Hydrostatic

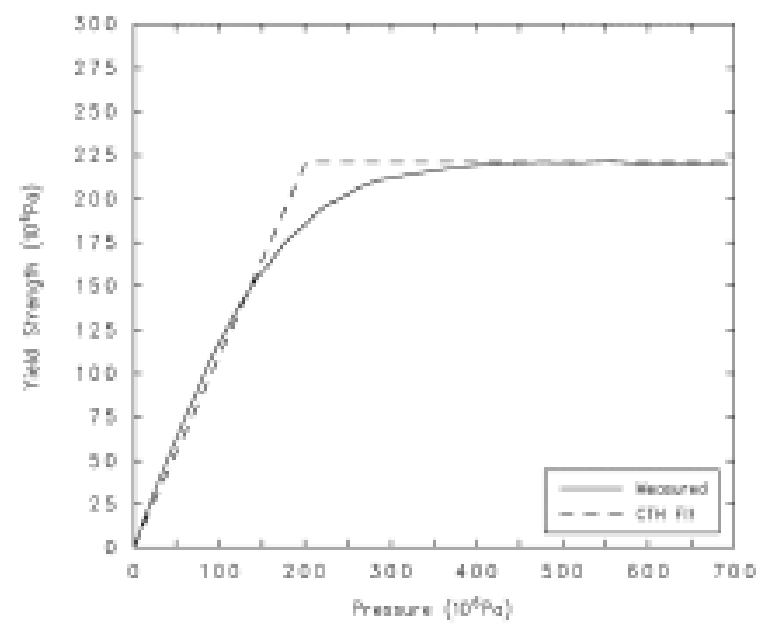

(b) Yield Envelope

Fig. 9. Material response for sand.

\section{Analysis of Test 3}

The problem setup for Test 3 is identical to that for Test 1, with the following exceptions: (1) the test bed was filled with compacted concrete sand, and (2) the unconfined compressive strength of the slab was $40.4 \mathrm{MPa}$. In the CTH problem setup, the test bed region was modeled as sand with the surrounding in-situ soil modeled as clay. As with the Test 1 analysis, the response for the sand was modeled using a P-alpha EOS and GEO strength model. The CTH material model driver was used to develop an initial fit to the static uni-axial strain and tri-axial compression data reported by Baylot [3]. Comparisons were then drawn with the measured free-field impulse and velocity data, with the model calibrated as needed to provide better correlation with the data. Figure 9 provides comparisons of the CTH-developed fits and those reported by Baylot [3].

The model calibration to the free-field data was problematic. The initial fit to the static material data provided a starting point for model calibration to the free-field data. Variations in model inputs for both the P-alpha EOS and GEO model were considered to improve correlation with the free-field data. In general, the "best" fit shown in Fig. 9 led to good correlation with the measured initial peak pressure and velocity at all gage ranges; however, there was generally poor correlation with the time-of-arrival (TOA) and impulse. This is illustrated in Fig. 10, where comparisons are provided for the free-field impulse and velocity at a range of $1.52 \mathrm{~m}$ from the charge. The TOA was consistently under-predicted at all gage locations. The impulse was under-predicted (on the order of 30 percent) at ranges less than or equal to $1.52 \mathrm{~m}$. At the larger ranges of 1.82 and $2.13 \mathrm{~m}$, there was reasonable correlation with the measured data (within 10 percent). The under-predicted impulse is a consequence of under-predicting the duration of the pressure pulse (there was excellent correlation with the peak pressures). During the course of model calibration, numerous attempts were made to increase the pulse width without success.

The P-alpha EOS and GEO strength models have limitations. First, the P-alpha EOS does not modify the material porosity during unloading. Second, and perhaps more importantly, the two models operate independently. Thus, changes in porosity have no influence on the material yield. Sand is both highly porous and has a high shear-strength, even at low confining pressures. It is apparent the model limitations affected the robustness of the constitutive modeling. These limitations were not an issue for the clay since it has low porosity and negligible shear-strength. There is clearly a need to investigate alternate constitutive models within CTH for modeling sandy soils and one should view this calibrated model as only a very coarse approximation for the dynamic soil response.

The calculated damage to the RC slab could be characterized as light and is consistent with that observed in the test. Comparisons of the structure velocity at AHS-0 and AHS-10 are provided in Fig. 11. These results are indicative of comparisons at other gage locations. There is reasonably good agreement with the measured peak 


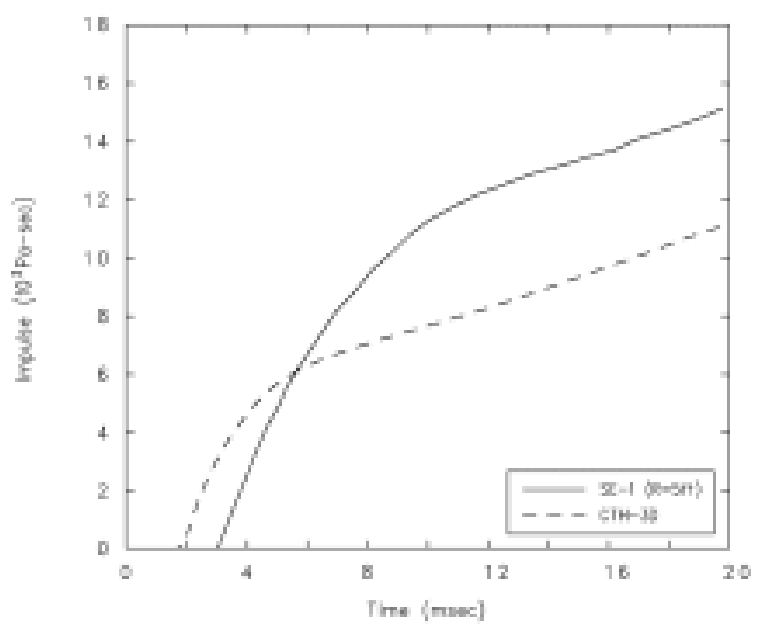

(a) Impulse

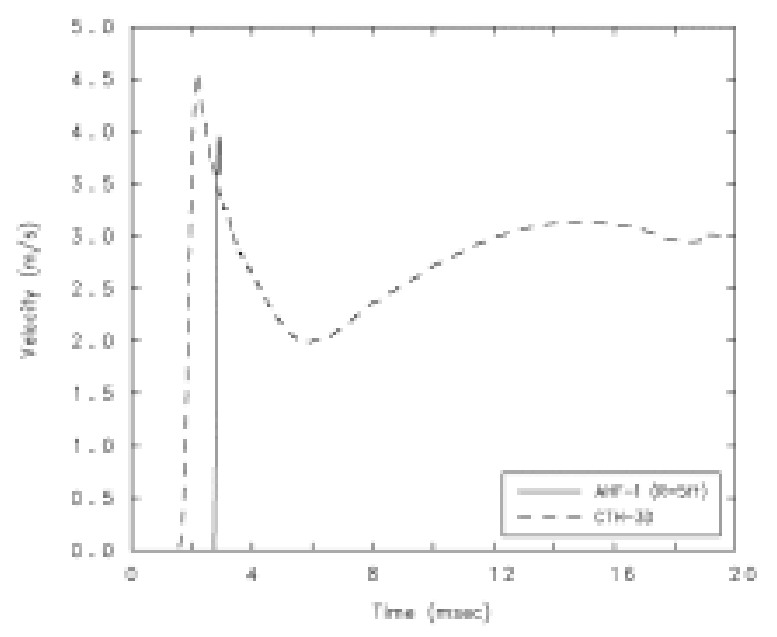

(b) Velocity

Fig. 10. Comparisons of free-field impulse and velocity at a range of $1.52 \mathrm{~m}$, test 3 .

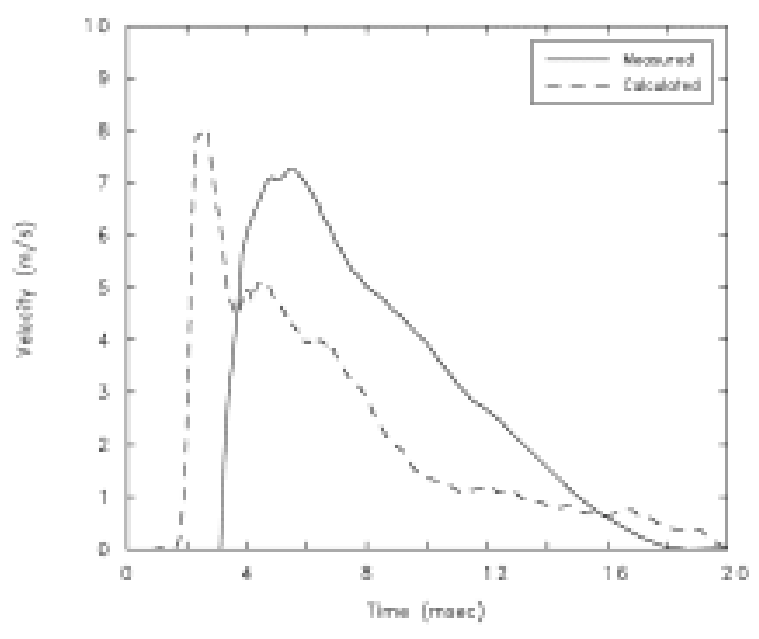

(a) AHS-0

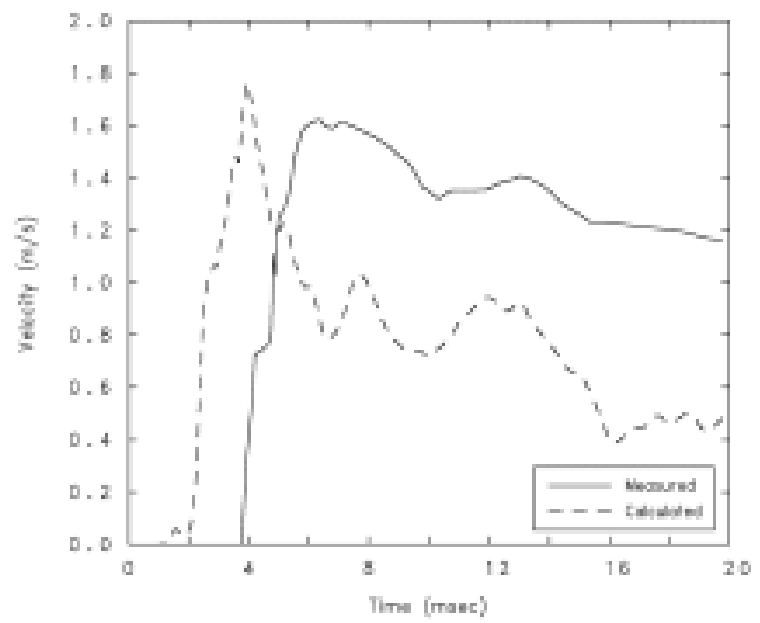

(b) AHS-10

Fig. 11. Velocity at accelerometer locations AHS-0 and AHS-10, test 3.

velocities; however, there are noticeable differences later in time. In general, it appears there is a much faster decay from the initial velocity as compared with that measured. Also, as noted with the free-field comparisons, the calculated TOA is much shorter than that measured. The disparities are attributed to inaccuracies in soil modeling. Given the tendency to under-predict the pressure pulse width in the free-field, one can conjecture that the duration of loading on the structure is under-predicted as well. This is consistent with the velocity history, where a sharper decay is noted in the calculation. Comparisons for the reaction structure indicate less correlation with the measured data as compared with previous calculations. In particular, there are numerous oscillations in the translational velocity. The reason for these oscillations is unclear, but is likely attributed to mesh effects and/or inaccuracies in the soil modeling.

As an aside, it is interesting to compare results from Tests 1 and 3. The setups for the two tests are virtually identical, with the only major difference being the backfill media in the test bed. The high shock transmission 
qualities of the clay resulted in larger loads on the structure, which eventually led to breach of the RC slab. In contrast, only light damage occurred to the structure when it was embedded in sand. Qualitatively, the coupling algorithm was able to capture the influence of backfill media on the blast/structure interaction (shortcomings with modeling the sand response preclude reliable quantitative comparisons). Another interesting point is that the coupling algorithm was capable of modeling the two distinct soil regions in the Test 3 benchmark, i.e., the test bed composed of sand and the surrounding in-situ clay. The complexity of the problem setup, which is typical of real-world applications, can only be modeled using a physics-based approach (i.e., via FE methods, CEL, Particle-methods, etc.). Furthermore, modeling blast loading on buried structures generally requires a fully coupled analysis as one can expect large structure deformations and long load duration (at least, relative to what is encountered in air blast applications).

\section{Conclusions}

CEL methods are well suited for modeling blast loading on buried structures. The flexibility in choosing which portions of the problem are modeled as Lagrangian or Eulerian is the method's greatest attribute. However, the accuracy of the CEL solution is highly contingent on the data provided for the different computational domains. For the CONWEB benchmarks, the data that most influenced the analysis were the constitutive modeling of the backfill media. This was most evident in the Test 3 benchmark, where limitations associated with using the P-alpha EOS and GEO strength model were found to affect modeling of the dynamic response for sand. These models, on the other hand, worked well for the clay. Thus, a future direction for research is to investigate alternate constitutive models within CTH for modeling sandy soils (or any soil exhibiting high porosity and/or a high shear strength at low confining pressures).

\section{Acknowledgements}

The author would like to thank Dr. James Baylot (ERDC) for providing experimental data along with his insights. Sandia is a multi-program laboratory operated by Sandia Corporation, a Lockheed Martin Company, for the United States Department of Energy under Contract DE-AC04-94AL85000.

\section{References}

[1] S.W. Attaway, F.J. Mello, M.W. Heinstein, J.W. Swegle, J.A. Ratner and R.I. Zadoks, Pronto3D Users Instructions: Transient Dynamic Code for Nonlinear Structural Analysis, Sandia National Laboratories Report SAND98-1361, 1998.

[2] S.W. Attaway, R.V. Matalucci, S.W. Key, K.B. Morrill, L.J. Malvar and J.E. Crawford, Enhancements to Pronto3D to Predict Structural Response to Blast, Sandia National Laboratories Report SAND2000-1017, 2000.

[3] J.T. Baylot, Parameters Affecting Loads on Buried Structures Subjected to Localized Blast Effects, Waterways Experiment Station Report TR-SL-92-9, 1992.

[4] G.C. Bessette, Modeling Coupled Blast/Structure Interaction with Zapotec, Benchmark Calculations for the Conventional Weapon Effects Backfill (CONWEB) Tests, Sandia National Laboratories Report SAND2004-4096, 2004.

[5] R.L. Bell, M.R. Baer, R.M. Brannon, D.A. Crawford, M.G. Elrick, E.S. Hertel, S.A. Silling and P.A. Taylor, CTH User's Manual and Input Instructions, Version 6.0, Sandia National Laboratories Report, 2000.

[6] P.G. Hayes, Backfill Effects on Response of Buried Reinforced Concrete Slabs, Waterways Experiment Station Report TR-SL 89-19, 1989.

[7] H.U. Mair, Review: Hydrocodes for Structural Response to Underwater Explosions, Shock and Vibration 6 (1999), 81-96.

[8] L.J. Malvar, J.E. Crawford, J.W. Wesevich and D. Simons, A Plasticity Concrete Material Model for DYNA3D, International Journal of Impact Engineering 19 (1997), 847-873. 

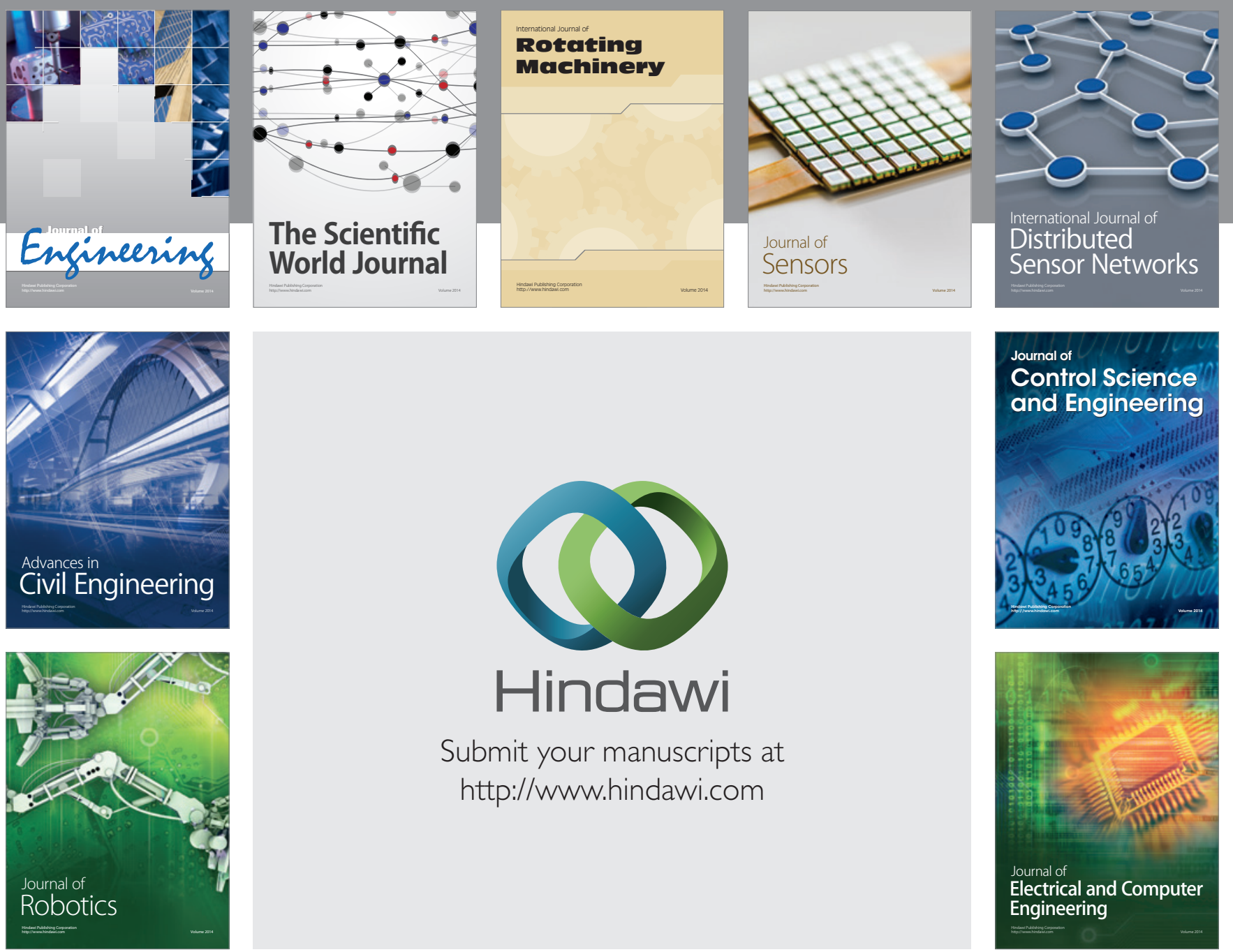

Submit your manuscripts at

http://www.hindawi.com
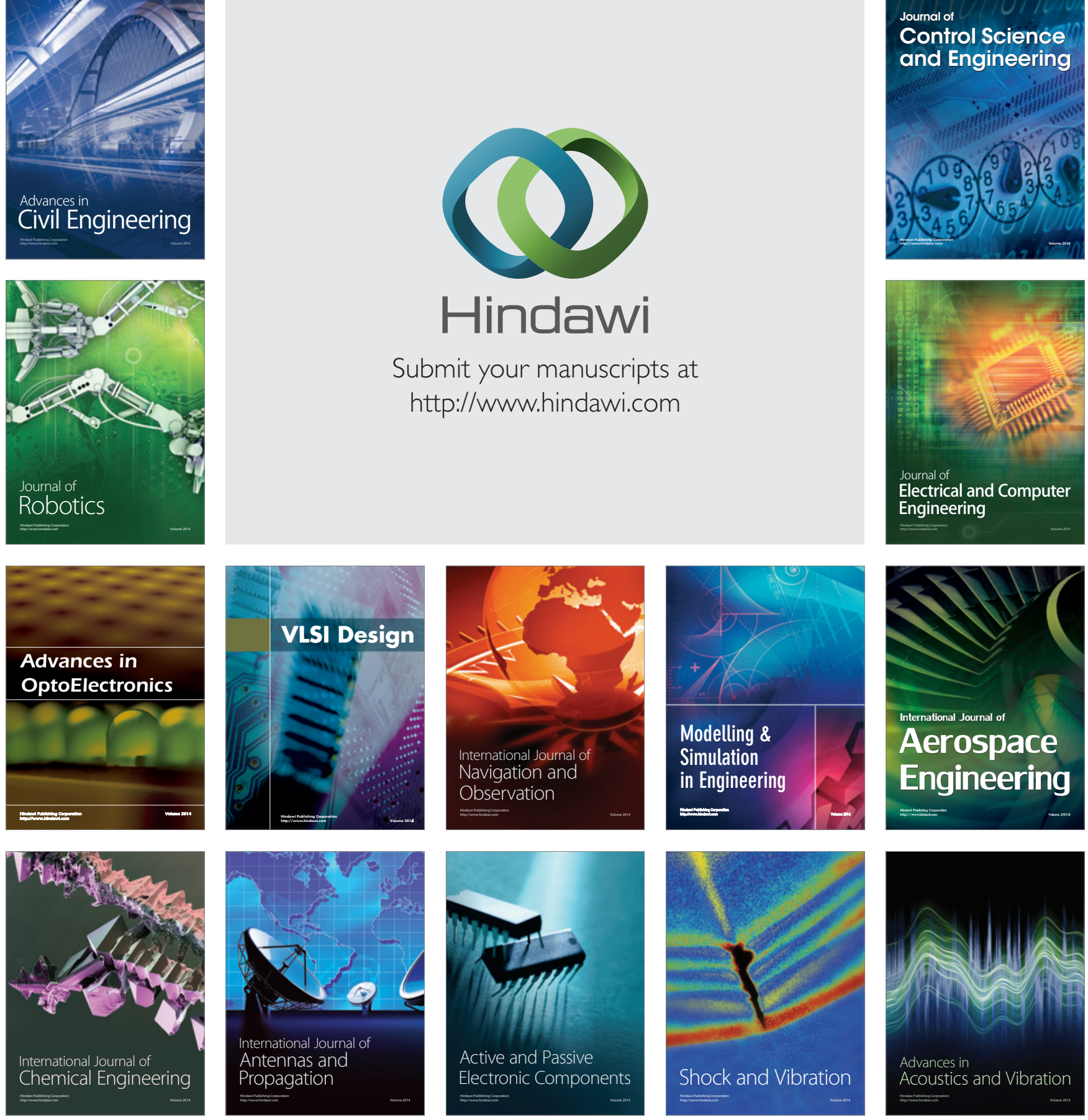